\title{
Cosmological test of gravity with gravitational waves
}

\author{
Atsushi Nishizawa ${ }^{1}$ \\ Research Center for the Early Universe (RESCEU), School of Science, The University of Tokyo \\ 7-3-1 Hongo, Bunkyo-ku, Tokyo 113-0033, Japan \\ E-mail: anishieresceu.s.u-tokyo.ac.jp
}

The direct detection of gravitational waves (GWs) from merging binary black holes and neutron stars mark the beginning of a new era in gravitational physics, and it brings forth new opportunities to test theories of gravity. The properties of GW propagation are modified in alternative theories of gravity and are crucial observables to test gravity at cosmological distance. From the coincident detection of GW170817/GRB170817A, the propagation speed has already been measured so precisely that a class of gravity theories in which GWs propagate with the speed different from $\mathrm{c}$ has been ruled out. On the other hand, another conspicuous feature in modified gravity is the time variation of gravitational constant $(\mathrm{G})$, which affects the amplitude damping of GWs. The constraint on the time variation of $\mathrm{G}$ for $\mathrm{GWs}$ has obtained from GW170817/GRB170817A but is still too weak. To deal with the current situation of testing gravity with GWs, we proposed a new universal framework for testing gravity with GWs, based on the generalized propagation of a GW in an effective field theory that describes modification of gravity at cosmological scales. Then we performed a parameter estimation study, showing that the future observation of GW can constrain the parameter of the amplitude damping in the generalized models of GW propagation down to a level of $1 \%$. We also studied the time variation of the gravitational couplings in Horndeski theory by performing Monte Carlo-based numerical simulations. From the simulation results, we find that the current accelerating Universe prefers the models with less damping of GWs and that almost all models produced in the numerical simulation can be tested by the future GW observation. In this article, we review GW propagation test of gravity and its implication for cosmology, particularly focusing on our recent works.

The 4th KMI International Symposium (KMI2019)

18-20, February 2019

Nagoya, Japan 


\section{Introduction}

To explain the origin of the accelerating expansion of the Universe at present, a possibility to modify gravity theories at cosmological distance has been proposed [1-5]. If gravity strength is modified, it can affect the cosmological observables such as the cosmic microwave background and the large-scale structure (see [6] for a review). At the same time, the modification of gravity changes the properties of gravitational-wave $(\mathrm{GW})$ propagation as well $[7,8]$. Particularly, GW propagation can feel the cosmic expansion, that is, gravity at cosmological distance. Therefore, searching for deviations from general relativity during the propagation of a GW is also crucial for testing gravity at cosmological scales and constraining modified gravity theories which accelerate the cosmic expansion.

To this end, it is crucial to search for anomalous deviations from general relativity (GR) in a model-independent way. One of such model-independent tests is measuring the propagation speed of a GW [9]. In GR, a GW propagates with the speed of light, while in an alternative theories of gravity the propagation speed could deviate from the speed of light due to the modification of gravity and quantum effects of spacetime. Before the occurrence of the coincidence event, GW170817/GRB170817A [10], it had been expected that comparing arrival times between GW from binary neutron stars (BNS) merger and high-energy photons from a short gamma-ray burst emitted almost at the same time can measure GW propagation speed at a precision of $10^{-16}$

$10^{-15}[11,12]$ and consequently tightly constrain the modification of gravity relevant to the cosmic accelerating expansion [13]. One of the other model-independent tests is to check the existence of GW polarization modes predicted in GR and to search for additional polarizations [14]. In GR, a GW has two polarizations, while there could be at most four additional (two scalar and two vector) polarizations in alternative theories of gravity. With multiple detectors, it is possible to detect the additional modes by separating them $[15,16]$ or constructing a null signal $[17,18]$.

From the GW data of binary black holes $(\mathrm{BBH})$ detected by aLIGO, the constraints have been obtained on graviton mass to be $\mathrm{m}_{\mathrm{g}} \approx 7.7 \times 10^{-23} \mathrm{eV}$ [19] and on the modified dispersion relation $[19,20]$. More recently, the triple detector network of aLIGO and VIRGO has explored additional polarizations and given upper limits on their existence [21-23]. The coincidence detection of GW 170817 and GRB170817A [10] brought us the first opportunity to measure the speed of a GW from the arrival time difference and constrained the deviation from the speed of light at the level of $10^{-15}$ [24]. Consequently, from this constraint, the strong limit on gravity modifications relevant to the current accelerating expansion of the Universe has been obtained [25-29].

Other than the modification on the propagation speed, the variation of gravitational constants is one of the prominent signatures of modified gravity. In modified gravity theories, there appear in general multiplicate gravitational couplings for the Poisson equation $\mathrm{G}_{\text {matter, }}$, the gravitational lensing equation $\mathrm{G}_{\text {light }}$, and $\mathrm{GWs}_{\mathrm{gw}}[30,31]$. Particularly, $\mathrm{G}_{\mathrm{gw}}$ affects the amplitude of a GW [32-34]. Therefore, measuring the gravitational couplings at different times by multiple tracers and testing the equivalence to the Newton constant are a crucial direction in the next decade to pin down a correct theory of gravity at cosmological scales. In this article, we review GW propagation test of gravity and its implication for cosmology, particularly focusing on our recent works.

\section{Universal framework for testing gravity with $\mathrm{GW}$ propagation}

Following the general formulation of GW propagation in an effective field theory [7], tensor perturbations obey the equation of motion

$$
h_{i j}^{\prime \prime}+(2+\nu) \mathcal{H} h_{i j}^{\prime}+\left(c_{T}^{2} k^{2}+a^{2} \mu^{2}\right) h_{i j}=a^{2} \Gamma \gamma_{i j},
$$

where the prime is a derivative with respect to conformal time, a is the scale factor, $\mathcal{H} \equiv \mathrm{a}^{\prime} / \mathrm{a}$ is the Hubble parameter in conformal time, $v=\mathcal{H}^{-1}\left(\mathrm{~d} \ln \mathrm{M}^{*} / \mathrm{dt}\right)$ is the Planck mass run rate, $\mathrm{c}_{\mathrm{T}}$ is the GW propagation speed, and $\mu$ is graviton mass. The source term $\Gamma \gamma_{\mathrm{ij}}$ arises from anisotropic 
stress. In the limit of $\mathrm{c}_{\mathrm{T}}=1$ and $\nu=\mu=\Gamma=0$, the propagation equation (1) is reduced to the standard one in GR. At a linear level or large scales in the Friedmann-Lemaitre-RobertsonWalker (FLRW) background, the modification functions are simply functions of time [7].

Here we focus on modifications of gravity as an explanation for the cosmic accelerating expansion and on observations by ground-based GW detectors. In other words, all the modification functions in Eq.(1) are slowly varying functions with cosmological time scale, while GW wavelength $\sim \mathrm{k}^{-1}$ is much smaller than the cosmological horizon scale. Thus, we can obtain a WKB solution for Eq.(1). The WKB solution in the absence of the source term $(\Gamma=0)$ in the generalized GW propagation (gGP) framework is [8]:

$$
\begin{aligned}
h & =e^{-\mathcal{D}} e^{-i k \Delta T} h_{\mathrm{GR}}, \\
\mathcal{D} & =\frac{1}{2} \int_{0}^{z} \frac{\nu}{1+z^{\prime}} d z^{\prime}, \\
\Delta T & =\int_{0}^{z} \frac{1}{\mathcal{H}}\left(\frac{\delta_{g}}{1+z^{\prime}}-\frac{\mu^{2}}{2 k^{2}\left(1+z^{\prime}\right)^{3}}\right) d z^{\prime} .
\end{aligned}
$$

where $\mathcal{D}$ is the damping factor, and $\Delta \mathrm{T}$ is the time delay due to the effective GW speed different from speed of light, and we defined $\delta_{\mathrm{g}}=1-\mathrm{c}_{\mathrm{T}}$ as a tiny parameter. The gGP framework is quite general and can be applied to many theories of modified gravity such as Horndeski theory with nonzero $v$ and $\delta_{\mathrm{g}}$, including $\mathrm{f}(\mathrm{R})$ gravity with nonzero $v$ as a special case, and Einstein-aether theory with nonzero $\delta_{\mathrm{g}}$.

\section{Future constraint from GW observations}

In this section, we investigate with a Fisher information matrix how precisely we can measure the model parameters in future GW observations. For the complete description of the parameter estimation method with the Fisher matrix, see [8].

\subsection{Numerical setup}

We consider the simplest waveform in which $v$ and $\mu$ are assumed to be constant and $\delta_{\mathrm{g}}$ and $\Gamma$ are zero. Setting $\delta_{\mathrm{g}}=0$ is motivated by the recent measurement of the GW speed from GW170817/GRB170817A [10,24,35]. $\Gamma=0$ is just for simplicity, but is true in most gravity theories including the Horndeski theory. Under these assumptions, the waveform in Eqs.(2)-(4) is reduced to

$$
\begin{aligned}
h & =(1+z)^{-\nu / 2} e^{-i k \Delta T} h_{\mathrm{GR}}, \\
\Delta T & =-\frac{\mu^{2}}{2 k^{2}} \int_{0}^{z} \frac{d z^{\prime}}{\left(1+z^{\prime}\right)^{3} \mathcal{H}} .
\end{aligned}
$$

For the GR waveform $h_{\mathrm{GR}}$, we will use the phenomenological waveform (PhenomD) [36] (compiled in Appendix of [8]), which is an up-to-date version of inspiral-merger-ringdown (IMR) waveform for aligned-spinning (nonprecessing) binary black holes (BBH). While for BH-NS binaries and BNS, we will use the inspiral waveform up to $3.5 \mathrm{PN}$ order in phase, which is an early inspiral part of the PhenomD waveform. The waveform in Eq.(5) has in total 13 parameters: the redshifted chirp mass, the symmetric mass ratio, time and phase at coalescence, $t_{c}$ and $\phi_{\mathrm{c}}$, redshift $\mathrm{z}$, symmetric and asymmetric spins, $\chi_{\mathrm{s}}$ and $\chi_{\mathrm{a}}$, the angle of orbital angular momentum measured from the line of sight $\iota$, sky direction angles of a source $\left(\theta_{\mathrm{S}}, \phi_{\mathrm{S}}\right)$, polarization angle $\psi$, and gravitational modification parameters, $v$ and $\mu$. We will assume the flat $\Lambda$-Cold-DarkMatter $(\Lambda \mathrm{CDM})$ model and fix cosmological parameters to those determined by Planck satellite [37]. Then the luminosity distance $d_{L}$ is mapped into redshift $\mathrm{z}$ by the standard formula of the luminosity distance for photons. On the other hand, as seen from Eq.(5), the effective luminosity distance for GWs is $\mathrm{d}_{\mathrm{L}}{ }^{\mathrm{gw}}=(1+\mathrm{z})^{-\mathrm{v} / 2} \mathrm{~d}_{\mathrm{L}}(\mathrm{z})$. Since the distance measured by a GW is $\mathrm{d}_{\mathrm{L}}{ }^{\mathrm{gw}}$, $\mathrm{z}$ and $v$ are completely degenerated. Therefore, we need source redshift information by identifying a host galaxy or detecting an electromagnetic transient counterpart. Redshift information would 
be available even for $\mathrm{BH}$ binaries only if they are located at low redshift, $\mathrm{z}<0.1$, and have high SNR or good angular resolution so that a unique host galaxy is identified [38,39].

The forecast constraints from the planned GW detectors are estimated with the Fisher information matrix. We consider here a detector network composed of aLIGO at Hanford and Livingston, and aVIRGO (HLV) as the second-generation (2G) detectors and Voyager, ET, and CE as the third-generation $(3 \mathrm{G})$ detectors. The $3 \mathrm{G}$ detectors are much more sensitive at lower frequencies and can start observing GWs from compact binaries much earlier than the second-generation detectors. For BNS, it is $\sim 2$ hours and $\sim 1$ day before merger at $5 \mathrm{~Hz}$ and $2 \mathrm{~Hz}$, respectively. Even a BH-NS binary takes several hours before merger below $3 \mathrm{~Hz}$. This is a merit for detectors because the Earth rotation during observing a signal allows the detector response functions to change their directions and improve the sky localization even with less number of detectors [40]. Therefore, we take into account the time evolution of the detector response functions correctly for $3 \mathrm{G}$ detectors in the Fisher matrix analysis.

In the following analysis, we will set fiducial parameters, $\mathrm{t}_{\mathrm{c}}, \phi_{\mathrm{c}}, \chi_{\mathrm{s}}, \chi_{\mathrm{a}}, v$, and $\mu$ to zero and randomly generate sky locations $\left(\theta_{\mathrm{S}}, \phi_{\mathrm{S}}\right)$ and other angle parameters $(\iota, \psi)$ for compact binaries with fixed masses and redshift. In the procedure of the source generation, we set the network signal-to-noise ratio (SNR) threshold for detection to $\rho=8$ and keep only sources with $\rho>8$.

\subsection{Parameter estimation errors}

We generated 500 sources for each class of compact binaries: $30 \mathrm{M}_{\text {sun }}-30 \mathrm{M}_{\text {sun }} \mathrm{BBH}, 10$ $\mathrm{M}_{\text {sun }}-10 \mathrm{M}_{\text {sun }} \mathrm{BBH}, 10 \mathrm{M}_{\text {sun }} \mathrm{BH}-1.4 \mathrm{M}_{\text {sun }} \mathrm{NS}$, and 1.4 $\mathrm{M}_{\text {sun }}-1.4 \mathrm{M}_{\text {sun }} \mathrm{BNS}$, at $\mathrm{z}=0.05$ for the HLV detector network and at $\mathrm{z}=0.1$ and 0.5 for the $3 \mathrm{G}$ detector networks. Heavier sources give a tighter constraint on $v$. Among the sources we studied, $30 \mathrm{M}_{\text {sun }}-30 \mathrm{M}_{\text {sun }} \mathrm{BBH}$ is the best source to measure $v$, irrespective of a source redshift. With the $2 \mathrm{G}$ detectors, we can achieve the measurement of $v$ up to at a level of $\Delta v \approx 1.3$ by observing a single source. However, seen in the GW propagation equation (1), the $v$ error of 1.3 is not good enough to constrain modified gravity because it should be compared with 2 . While the detector networks of 3 Voyager, 2 ET-D, and $3 \mathrm{CE}$ (or $3 \mathrm{ET}$-D) can reach $\Delta v \approx 0.3,0.04$, and 0.02 , respectively, with a single GW event among top $1 \%$ events. On the other hand, $2 \mathrm{CE}$ cannot improve the error because of poor sky localization.

\section{Application to Horndeski theory}

\subsection{Horndeski theory after GW170817}

A coincidence event between GW from a NS binary merger and a short gamma-ray burst, GW170817/GRB170817A [10] gave the extremely tight constraint on the constant propagation speed of a GW, $-7 \times 10^{-16}<\delta_{\mathrm{g}}<3 \times 10^{-15}$ [24]. Consequently, gravity modifications relevant to the current accelerating expansion of the Universe has been limited tightly [25-29]. From the observational requirement for the propagation speed, the Lagrangian of the Horndeski theory [41-43] is reduced to the simple one [25-29]

$$
\mathcal{L}=G_{2}(\phi, X)-G_{3}(\phi, X) \square \phi+G_{4}(\phi) R .
$$

Here $\mathrm{X}=-\phi_{\mu} \phi^{\mu} / 2$, the canonical kinetic energy density of $\phi$.

\subsection{Gravitational couplings in Horndeski theory}

Let us assume the flat FLRW Universe. Under the quasi-static approximation, we ignore all dynamical terms in the equations of motion and derive the Poisson and lensing equations $[30,31]$

$$
\begin{aligned}
k^{2} \Psi & \simeq-4 \pi G_{\text {matter }}(k, \tau) \delta \rho_{\mathrm{m}}, \\
k^{2}(\Psi+\Phi) & \simeq-8 \pi G_{\text {light }}(k, \tau) \delta \rho_{\mathrm{m}},
\end{aligned}
$$


where $\tau$ is the conformal time, $\delta \rho_{\mathrm{m}}$ is the density fluctuation of matter. $\mathrm{G}_{\text {matter }}$ and $\mathrm{G}_{\text {light }}$ denote the effective gravitational couplings for matter clustering and gravitational lensing, respectively. In modified gravity theories, in general $\mathrm{G}_{\text {matter }} \neq \mathrm{G}_{\text {light, }}$ i. e., gravitational potentials, $\Psi$ and $\Phi$, are no longer equivalent each other, leading to the violation of the equivalence principle. Moreover, the values of $\mathrm{G}_{\text {matter }}$ and $\mathrm{G}_{\text {light }}$ also can deviate from the Newton constant $\mathrm{G}_{\mathrm{N}}$.

For the specific case of the Horndeski theory in Eq.(7), gravitational couplings at cosmological scales $[44,45]$ are

$$
G_{\text {matter }}=G_{\text {light }}=G_{N} \frac{M_{\mathrm{pl}}^{2}}{M_{*}^{2}},
$$

in the super-Compton case and

$$
\begin{aligned}
& G_{\text {matter }}=G_{N} \frac{M_{\mathrm{pl}}^{2}}{M_{*}^{2}}\left(1+\beta_{\xi}^{2}\right), \\
& G_{\text {light }}=G_{N} \frac{M_{\mathrm{pl}}^{2}}{M_{*}^{2}}\left[1+\beta_{\xi}^{2}+\sqrt{\frac{2}{c_{S}^{2} D}} \frac{\alpha_{M} \beta_{\xi}}{2}\right],
\end{aligned}
$$

in the sub-Compton case, where $\mathrm{M}^{2}{ }^{2}=2 \mathrm{G}_{4}$ and

$$
\alpha_{M}=\frac{1}{H} \frac{d \log M_{*}^{2}}{d t} .
$$

Here and hereafter we use $\mathrm{M}_{\mathrm{pl}}$ to denote the reduced Planck mass. The other functions, $\beta \xi, \mathrm{D}$, and $\mathrm{c}_{\mathrm{s}}{ }^{2}$, are the functions dependent on the time evolution of a scalar field [46]. Particularly, the function $\beta_{\xi}$ plays an important role to distinguish the gravitational couplings. This difference is originated from the fluctuations of a scalar field. The function $\alpha_{M}$ in the Horndeski theory is exactly equivalent to $v$ in the gGP framework.

Substituting Eq.(13) into Eq.(3), we can express the amplitude modification in terms of the gravitational constant as

$$
e^{-\mathcal{D}}=\frac{M_{*}(z)}{M_{*}(0)}=\sqrt{\frac{G_{\mathrm{gw}}(0)}{G_{\mathrm{gw}}(z)}} .
$$

Here we defined $\mathrm{G}_{\mathrm{gw}} \equiv \mathrm{G}_{\mathrm{N}} \mathrm{M}_{\mathrm{pl}}{ }^{2} / \mathrm{M}^{2}$. Since the GR waveform is inversely proportional to the luminosity distance, one can interpret the amplitude modification as a correction to the electromagnetic luminosity distance $\mathrm{d}_{\mathrm{L}}(\mathrm{z})$, defining the effective luminosity distance for GWs $[32,33]$

$$
d_{\mathrm{L}}^{\mathrm{gw}} \equiv e^{\mathcal{D}} d_{\mathrm{L}}(z)=\sqrt{\frac{G_{\mathrm{gw}}(z)}{G_{\mathrm{gw}}(0)}} d_{\mathrm{L}}(z),
$$

\subsection{Numerical model sampling}

To assess the impact of the $v$ measurement on the Horndeski theory, we compute the time evolutions of $\alpha_{\mathrm{M}}, \mathrm{G}_{\mathrm{matter}}$, and $\mathrm{G}_{\text {light }}$ with the numerical method we developed previously in [29]. In this method of numerical model sampling, we expand the amplitude of a scalar field in power of time and also arbitrary functions in the Horndeski theory in power of $\phi$ and X. Their expansion coefficients are randomly given in the range of $[-1,1]$ by appropriately normalizing the overall magnitude. In the process of producing models above, we do not solve the equations of motion. To check the validity of models, we filter them with the two following criteria: (i) consistency with $\Lambda \mathrm{CDM}$ cosmology so that cosmological time evolution, $\mathrm{H}_{\mathrm{Horn}}$ and $\mathrm{dH}_{\mathrm{Horn}} / \mathrm{dt}$, coincides with $\mathrm{H}_{\Lambda \mathrm{CDM}}$ and $\mathrm{dH}_{\Lambda \mathrm{CDM}} / \mathrm{dt}$ within $20 \%$ observational errors [47], and (ii) stability of the theory avoiding ghost and gradient instabilities for the perturbations of scalar and tensor modes. We check the consistency conditions at specific redshifts: $\mathrm{z}=0,0.1,0.5,1.0,1.5$, and 2.0.

\subsection{Model distributions}

From Eqs.(10)-(12), we see $\mathrm{G}_{\text {matter }} \approx \mathrm{G}_{\text {light }}$ at the super-Compton scales, while $\mathrm{G}_{\text {light }}$ and $\mathrm{G}_{\text {matter }}$ are not equivalent at the sub-Compton scales. In addition to that, we are interested in how 
$\alpha_{\mathrm{M}}$ is distributed and related to $\mathrm{G}_{\text {matter }}$ and $\mathrm{G}_{\text {light. }}$. In Fig.1, we show $\alpha_{\mathrm{M}}$ in color on the $\mathrm{G}_{\text {light }}-$ $\mathrm{G}_{\mathrm{matter}}$ plane at different redshifts for two representative models of the Horndeski theory. At first glance, there is little difference between the Horndeski Lagrangian with/without the $\mathrm{G}_{3}$ function. This explicitly shows that the $\mathrm{G}_{3}$ term does not play any significant role to distribute models in the parameter space. At the super-Compton scales, all the models are aligned along the diagonal line, while at the sub-Compton scales, the off-diagonal scatter is apparent. This trend at subCompton scales is expected since the fluctuations of a scalar field become significant.
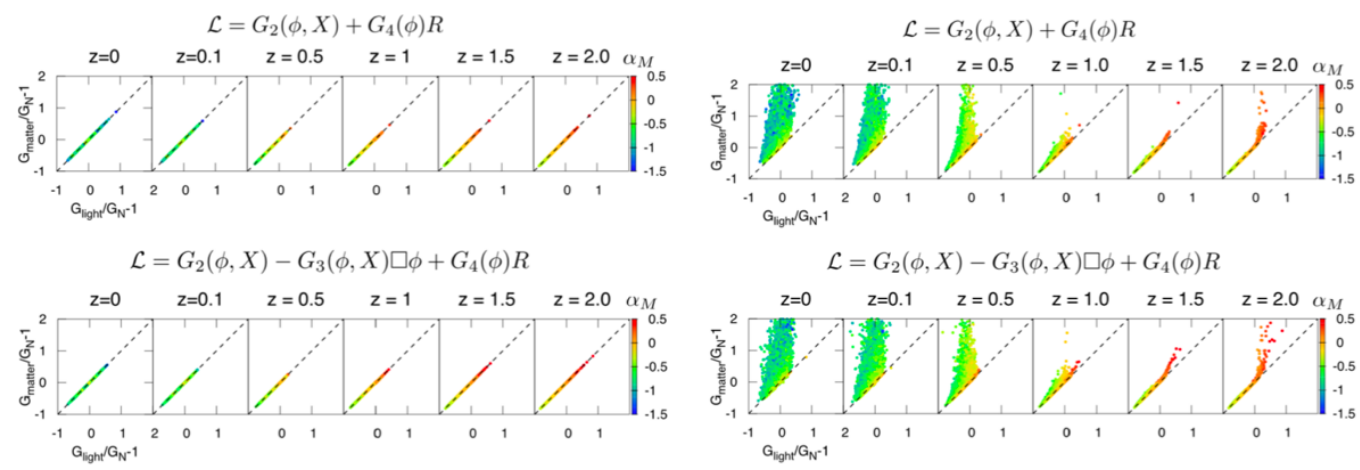

Figure 1. Time evolution of the correlation between $G_{\text {light }}$ and $G_{\text {matter }}$ at super-Compton limit (left panel) and at sub-Compton limit (right panel). The color bar shows the value of $\alpha_{M}$. The diagonal dashed lines show $G_{\text {matter }}=G_{\text {light }}$, i.e. $\Delta \gamma=0$. Top: models without $G_{3}$ term. Bottom: models with $G_{3}$ term. Reproduced from [29].

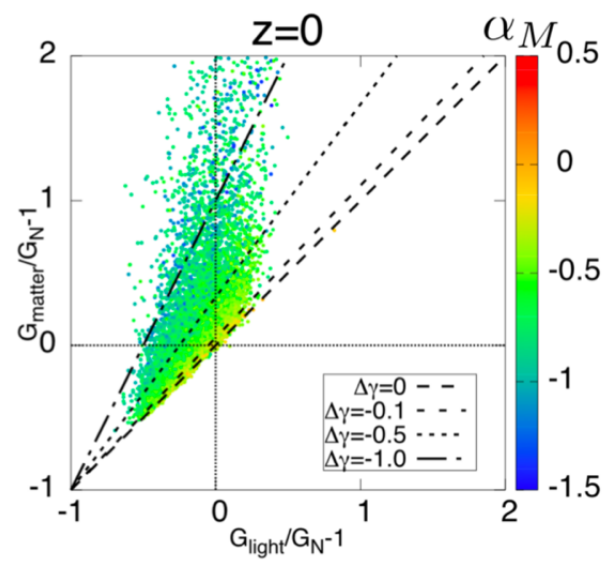

Figure 2. The contour plot of $\Delta \gamma$ on the $G_{\text {matter }}-G_{\text {light }}$ plane at $z=0$. Reproduced from [29].

\section{Current constraints and future prospect}

\subsection{Future GW observation}

The recent detection of GW170817 was accompanied with electromagnetic emissions in the broad range of electromagnetic frequencies and the redshift of the host galaxy was identified successfully. However, the constraint from GW170817 is too weak to test realistic models of modified gravity $(\Delta v \approx 80)$ [29]. The estimation errors of $v$ at a precision of $\Delta v \sim 1.3$ with the $2 \mathrm{G}$ detectors is still weak to test gravity at a precision comparable with other observations. Voyager and the $3 \mathrm{G}$ detectors such as $\mathrm{CE}$ and ET can reach the measurement error of $\Delta v \approx 0.02$.

For convenience, we introduce the deviation parameter $\Delta \gamma \equiv \gamma-1$, where $\gamma$ is the gravitational slip parameter [48-50] and is related to the gravitational couplings as $\mathrm{G}_{\text {light }} / \mathrm{G}_{\text {matter }}=1+\Delta \gamma$ 12. The gravitational slip parameter $\Delta \gamma$ is positively correlated with $\alpha_{\mathrm{M}}$, as we see in Fig.2. Observationally, the negativeness of $\alpha_{\mathrm{M}}$ is interesting because GW amplitude is enhanced than in 
general relativity, as seen from Eqs.(2)-(3). Since the possible range of $\alpha_{\mathrm{M}}$ parameter will be significantly constrained at the level of $\sim 0.01$ by the $3 \mathrm{G} \mathrm{GW}$ detectors, most models of the Horndeski theory with $\Delta \gamma<-0.01$ (most models we obtained numerically) will be tested. If we can measure on $v$ at the levels of $0.5,0.1$, and 0.02 and no deviation from GR is found, rejection fractions out of all models plotted in Fig.2 (corresponding lower limits on $\Delta \gamma$ ) are $65.37 \%$ (>1.5), $99.88 \%(>-0.031)$, and $99.99 \%\left(>-10^{-3}\right)$, respectively. We emphasize that positive $\alpha_{\mathrm{M}}$ and $\Delta \gamma$ are hardly realized in our numerical model sampling.

\subsection{Local measurements}

Although in the model of the Horndeski theory in Eq. (7), the Vainshtein mechanism recovers the Newtonian law of gravity at small scales, whereas the time variation of the gravitational couplings are allowed at cosmological scales [51]. However, the direct measurements of the gravitational couplings with local astronomical objects can constrain the present value of $\alpha_{\mathrm{M}}$, denoted by $\alpha_{\mathrm{M} 0}$, by connecting a local solution of a scalar field to a cosmological solution. The observations of the binary pulsars [52] and the lunar laser ranging experiments [53] currently give the constraint, $\left|\alpha_{\mathrm{M} 0}\right|<0.02$ [51]. As we discussed in this paper, the GW observation can constrain $\alpha_{\mathrm{M}}$ at the order of 0.01 , which is comparable with the local measurements. Since the observation of GW propagation can measure modification of gravity at cosmological scales directly, the combination with the local measurements should play a significant role to check the consistency of a gravity theory over the wide ranges of space and time.

\subsection{Cosmological measurements}

Cosmological observations also put bounds on the gravitational couplings. Recently, data from the CMB observation by Planck [50] were analyzed jointly with the galaxy survey data [54] and the cosmic shear measurement data by KiDS and GAMA observations [55] and the current stringent bound on $\alpha_{\mathrm{M} 0},\left|\alpha_{\mathrm{M} 0}\right|<0.04$ has been obtained. However, in order to put the bounds, it is crucial to assume simple forms of the time evolutions for them. In this sense, the constraints are artificially tightened and are difficult to be compared with GW observations. Furthermore, the simple parametrization may be problematic in that it cannot cover the whole parameter space of the Horndeski theory, as shown by [56,57] that the stable region of the Horndeski theory significantly depends on the parametrization taken.

\section{Conclusions}

To treat tests of gravity with GW more exhaustively and intuitively, irrespective of the models of gravity theories, GW sources, and background spacetimes, we have proposed a new universal framework for testing gravity, based on the propagation equation of a GW in an effective field theory, which is characterized by amplitude damping, propagation speed, graviton mass, and a possible source term. Then we have studied with the Fisher information matrix how precisely the modification of gravity is measured with future GW observations. For the model with constant $v$ and $\mu$, the $3 \mathrm{G}$ detectors such as $\mathrm{CE}$ and ET can reach the measurement error of $\Delta v \approx 0.02$. In the latter part, we have studied the model distribution of the Horndeski theory with a numerical approach. We performed a Monte Carlo-based numerical simulation and computed $\mathrm{G}_{\text {matter }}, \mathrm{G}_{\text {light }}$, and $\alpha_{\mathrm{M}}$, and found that (i) $\mathrm{G}_{\text {matter }} \approx \mathrm{G}_{\text {light }}$ in the super-Compton case, while $\mathrm{G}_{\text {matter }} \geq$ $\mathrm{G}_{\text {light }}$ in the sub-Compton case, (ii) model-filtering conditions consistent with $\Lambda \mathrm{CDM}$ cosmology preferentially select the negative sign of $\alpha_{\mathrm{M}}$ at lower redshifts $\mathrm{z}<1$, indicating that the observed amplitude of a GW is relatively enhanced.

Summarizing the above results, the future GW observations can constrain $v$ or equivalently $\alpha_{\mathrm{M}}$ in the Horndeski theory at the precision of $\mathrm{O}(0.01)$, which is comparable with the local measurements such as the binary pulsars and the lunar laser ranging. Therefore, the GW observation combined with the local and cosmological measurements play a significant role to check the consistency of a gravity theory at cosmological distance. 
A.N. is supported by JSPS KAKENHI Grant Nos. JP17H06358 and JP18H04581.

\section{References}

[1] S. Tsujikawa, Lect. Notes Phys. 800, 99 (2010).

[2] S. Nojiri and S. D. Odintsov, Phys.Rept. 505, 59 (2011).

[3] T. Clifton, P. G. Ferreira, A. Padilla, and C. Skordis, Phys. Rept. 513, 1 (2012).

[4] A. Joyce, L. Lombriser, and F. Schmidt, Ann. Rev. Nucl. Part. Sci. 66, 95 (2016).

[5] S. Nojiri, S. D. Odintsov, and V. K. Oikonomou, Phys. Rept. 692, 1 (2017).

[6] M. Ishak, Living Rev. Rel. 22, 1 (2019).

[7] I. D. Saltas, I. Sawicki, L. Amendola, and M. Kunz, Phys. Rev. Lett. 113, 191101 (2014).

[8] A. Nishizawa, Phys. Rev. D97, 104037 (2018).

[9] C. M. Will, Living Rev. Rel. 17, 4 (2014).

[10] B. P. Abbott et al. (Virgo, LIGO Scientific Collaboration), Phys. Rev. Lett. 119, 161101 (2017).

[11] A. Nishizawa and T. Nakamura, Phys. Rev. D 90, 044048 (2014).

[12] A. Nishizawa, Phys. Rev. D93, 124036 (2016).

[13] L. Lombriser and A. Taylor, arXiv:1509.08458 (2015).

[14] D. M. Eardley, D. L. Lee, A. P. Lightman, R. V. Wagoner, and C. M. Will, Phys. Rev. Lett. 30, 884 (1973).

[15] A. Nishizawa, A. Taruya, K. Hayama, S. Kawamura, and M.-a. Sakagami, Phys.Rev. D79, 082002 (2009).

[16] K. Hayama and A. Nishizawa, Phys.Rev. D87, 062003 (2013).

[17] K. Chatziioannou, N. Yunes, and N. Cornish, Phys.Rev. D86, 022004 (2012).

[18] Y. Hagihara, N. Era, D. Iikawa, and H. Asada, Phys. Rev. D98, 064035 (2018).

[19] B. P. Abbott et al. (LIGO Scientific Collaboration), Phys. Rev. Lett. 118, 221101 (2017).

[20] N. Yunes, K. Yagi, and F. Pretorius, Phys. Rev. D94, 084002 (2016).

[21] B. P. Abbott et al. (Virgo, LIGO Scientific Collaboration), Phys. Rev. Lett. 119, 141101 (2017).

[22] B. P. Abbott et al. (Virgo, LIGO Scientific Collaboration), arXiv:1709.09203 (2017).

[23] Y. Hagihara, N. Era, D. Iikawa, A. Nishizawa, and H. Asada, (2019), arXiv:1904.02300 [gr-qc].

[24] B. P. Abbott et al. (Virgo, Fermi-GBM, INTEGRAL, LIGO), Astrophys. J. 848, L13 (2017).

[25] T. Baker, E. Bellini, P. G. Ferreira, M. Lagos, J. Noller, and I. Sawicki, Phys. Rev. Lett. 119, 251301 (2017).

[26] P. Creminelli and F. Vernizzi, Phys. Rev. Lett. 119, 251302 (2017).

[27] J. Sakstein and B. Jain, Phys. Rev. Lett. 119, 251303 (2017).

[28] J. M. Ezquiaga and M. Zumalacarregui, Phys. Rev. Lett. 119, 251304 (2017).

[29] S. Arai and A. Nishizawa, Phys. Rev. D97, 104038 (2018).

[30] Y.-S. Song, G.-B. Zhao, D. Bacon, K. Koyama, R. C. Nichol, and L. Pogosian, Phys. Rev. D84, 083523 (2011).

[31] A. Johnson, C. Blake, J. Dossett, J. Koda, D. Parkinson, and S. Joudaki, Mon. Not. Roy. Astron. Soc. 458, 2725 (2016).

[32] E. Belgacem, Y. Dirian, S. Foffa, and M. Maggiore, Phys. Rev. D97, 104066 (2018).

[33] L. Amendola, I. Sawicki, M. Kunz, and I. D. Saltas, JCAP 1808, 030 (2018).

[34] R. C. Nunes, M. E. S. Alves, and J. C. N. de Araujo, arXiv:1811.12760 (2018). 
[35] B. P. Abbott et al. (GROND, SALT Group, OzGrav, DFN, INTEGRAL, Virgo, Insight-Hxmt, MAXI Team, Fermi-LAT, J-GEM, RATIR, IceCube, CAASTRO, LWA, ePESSTO, GRAWITA, RIMAS, SKA South Africa/MeerKAT, H.E.S.S., 1M2H Team, IKI-GW Follow-up, Fermi GBM, Pi of Sky, DWF (Deeper Wider Faster Program), Dark Energy Survey, MASTER, AstroSat Cadmium Zinc Telluride Imager Team, Swift, Pierre Auger, ASKAP, VINROUGE, JAGWAR, Chandra Team at McGill Univer- sity, TTU-NRAO, GROWTH, AGILE Team, MWA, ATCA, AST3, TOROS, Pan-STARRS, NuSTAR, ATLAS Telescopes, BOOTES, CaltechNRAO, LIGO, High Time Resolution Universe Survey, Nordic Optical Telescope, Las Cumbres Observatory Group, TZAC Consortium, LOFAR, IPN, DLT40, Texas Tech University, HAWC, ANTARES, KU, Dark Energy Camera GW-EM, CALET, Euro VLBI Team, ALMA), Astrophys. J. 848, L12 (2017).

[36] S. Khan, S. Husa, M. Hannam, F. Ohme, M. Purrer, X. J. Forteza, and A. Bohe, Phys. Rev. D 93, 044007 (2016).

[37] P. A. R. Ade et al. (Planck collaboration), Astron. Astrophys. 594, A13 (2016).

[38] H.-Y. Chen and D. E. Holz, arXiv:1612.01471 (2016).

[39] A. Nishizawa, Phys. Rev. D96, 101303 (2017).

[40] W. Zhao and L. Wen, Phys. Rev. D97, 064031 (2018).

[41] G. W. Horndeski, Int. J. Theor. Phys. 10, 363 (1974).

[42] C. Deffayet, X. Gao, D. A. Steer, and G. Zahariade, Phys. Rev. D84, 064039 (2011).

[43] T. Kobayashi, M. Yamaguchi, and J. Yokoyama, Prog. Theor. Phys. 126, 511 (2011).

[44] A. De Felice, T. Kobayashi, and S. Tsujikawa, Phys. Lett. B706, 123 (2011).

[45] L. Pogosian and A. Silvestri, Phys. Rev. D94, 104014 (2016).

[46] A. Nishizawa and S. Arai, arXiv:1901.08249 (2019).

[47] O. Farooq, F. R. Madiyar, S. Crandall, and B. Ratra, Astrophys. J. 835, 26 (2017).

[48] W. Hu and I. Sawicki, Phys. Rev. D76, 104043 (2007).

[49] B. Jain and P. Zhang, Phys. Rev. D78, 063503 (2008).

[50] P. A. R. Ade et al. (Planck), Astron. Astrophys. 594, A14 (2016).

[51] R. Kimura, T. Kobayashi, and K. Yamamoto, Phys. Rev. D85, 024023 (2012).

[52] W. W. Zhu et al., Mon. Not. Roy. Astron. Soc. 482, 3249 (2019).

[53] J. G. Williams, S. G. Turyshev, and D. H. Boggs, Phys. Rev. Lett. 93, 261101 (2004).

[54] J. Noller and A. Nicola, arXiv:1811.12928 (2018).

[55] A. Spurio Mancini et al., arXiv:1901.03686 (2019).

[56] M. Denissenya and E. V. Linder, JCAP 1811, 010 (2018).

[57] C. D. Kreisch and E. Komatsu, JCAP 1812, 030 (2018). 